\title{
SnTe PHASE TRANSITION IN STRAINED SUPERLATTICES PbTe/SnTe
}

\author{
O.A. Mironov $a$, O.N. MaKarovskiI ${ }^{a}$, O.N. NAShCHEKINA ${ }^{b}$,

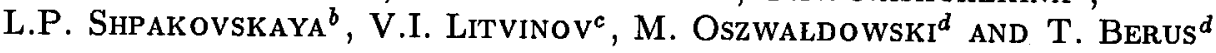 \\ a Institute of Radiophysics and Electronics, National Academy of Sciences of Ukraine \\ Kharkov, Ukraine \\ ${ }^{b}$ Polytechnical University of Kharkov; Kharkov, Ukraine \\ ${ }^{c}$ Institute of Material Science Problems, National Academy of Sciences of Ukraine \\ Chernovtsy, Ukraine \\ ${ }^{d}$ Institute of Physics, Poznań University of Technology \\ Piotrowo 3, 60-965 Poznan, Poland
}

An anomaly of the in-plane conductivity is observed in the superlattices $\mathrm{PbTe} / \mathrm{SnTe}$ on $(001) \mathrm{KCl}$ in the temperature region of $60-130 \mathrm{~K}$. The anomaly is caused by a structural phase transition in $\mathrm{SnTe}$ layer and as a result, the transition induced formation of defects. These defects are additional scattering centres which decrease the superlattice conductivity.

PACS numbers: $73.40 .-\mathrm{c}, 64.70 .-\mathrm{p}$

\section{Introduction}

The observation of superconductivity in $\mathrm{PbTe} / \mathrm{SnTe}$ superlattices (SL) [1] and its subsequent investigation [2-4] attracted attention to their galvanomagnetic properties.

It is known that on cooling down bulk single crystals of $\mathrm{SnTe}$, the compound undergoes a structural phase transition from cubic to rhombohedral phase (see review [5] and references therein). In the low temperature phase anion and cation sublattices are shifted relative to each other along one of the $\langle 111\rangle$-axes. The critical temperature $T_{\mathrm{c}}$ for bulk SnTe $(10-130 \mathrm{~K})$ depends on hole concentration, i.e. on the degree of deviation from ideal stoichiometry. The change in lattice symmetry of bulk single crystals SnTe is usually accompanied by weak and reversible (in respect of temperature change) anomalies, in the dependencies of electrical conductivity, with amplitude less than 1-2\%. PbTe single crystals also reveal the soft mode-type behaviour but phase transition has not been detected.

The main goal of the present study is the investigation of the phase transition in $\mathrm{PbTe} / \mathrm{SnTe}$ superlattices (SL's) by measurement of electrical conductivity and Hall coefficient $R_{\mathrm{H}}$. It is found that the displacing phase transition leads to irreversible changes in electrical properties of SL's under considerations. 


\section{Experimental results}

The strained $\mathrm{PbTe} / \mathrm{SnTe}$ SL's with misfit deformations on the interfaces are obtained by thermal evaporation in vacuum and alternate deposition of lead and tin telluride on $(001) \mathrm{KCl}$ and (111) $\mathrm{BaF}_{2}$ substrates. The SL's with period of 4, 12,16 , and $40 \mathrm{~nm}$ (the thicknesses of $\mathrm{PbTe}$ and $\mathrm{SnTe}$ layers are approximately equal) and period numbers from 12 up to 40 are grown. The periodicity of the $\mathrm{SL}$ is checked using X-ray diffraction (in $\mathrm{Cu} K_{\alpha}$ radiation), specifically by the appearance of the main reflection line $S_{0}$ from the "middle lattice" $\mathrm{PbTe} / \mathrm{SnTe}$ and satellites $S_{+n}$ of one or several orders located at equal distances on both sides of the main reflection line. The period of the "middle lattice" depends on $\mathrm{PbTe}$ and SnTe phase contents in multilayer. The SL period is calculated on the basis of distance between satellites.

The electrical conductivity $\sigma$ and Hall coefficient $R_{H}$ measurements are carried out using dc current conventional method on the double-Hall-cross-shaped samples in the temperature range of $4.2-300 \mathrm{~K}$ and magnetic fields of $0-1.5 \mathrm{~T}$.

The investigation of temperature dependencies of the electrical conductivity and Hall coefficient has shown that in the $\sigma(T)$ and $R_{\mathrm{H}}(T)$ dependencies there exist anomalies connected with the phase transition in SnTe layer in the vicinity of 100-200 K. In the same temperature region $\sigma$ and $R_{\mathrm{H}}$ anomalies are also observed in the individual $\mathrm{SnTe}$ layers. It is found that the anomalous character of the conductivity and Hall coefficient in the SL's depends essentially on preparation methods, the substrate orientation, SL period and also the number of cooling cycles. For instance, a maximum in the $\sigma(T)$ in the temperature range of 100-200 K is the most distinct in the first cycle of cooling down. Such a well pronounced maximum in $\sigma(T)$ is shown in Fig. 1 for a (001) oriented sample No. 354.

Repeated cycles of cooling lead to disappearance of the $\sigma(T)$ maximum. This change in the $\sigma(T)$ curve character, after the first cooling cycle, is accompanied

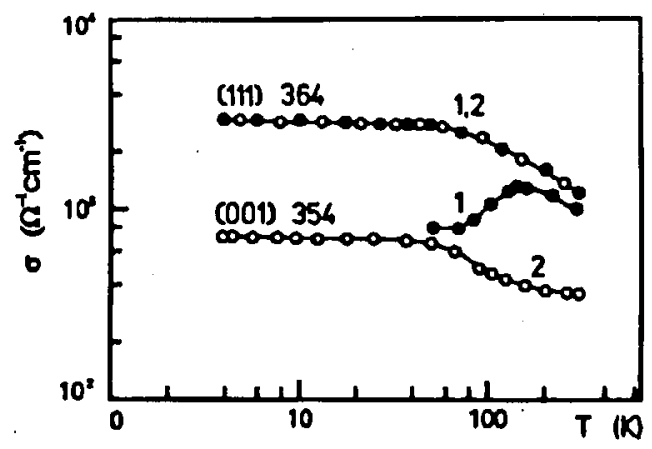

Fig. 1. Temperature dependence of the conductivity $\sigma$ for a (111) oriented sample 364 and $a(001)$ oriented sample 354. 1 and 2 are the first and the second warm-up's respectively. 


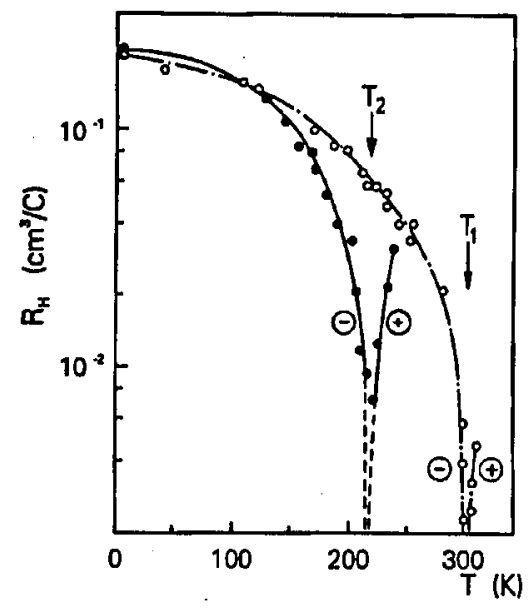

Fig. 2. Temperature dependence of the Hall coefficient $R_{\mathrm{H}}$ in sample $354 . T_{1}$ and $T_{2}$ are the temperatures of the $R_{\mathrm{H}}$ inversion. Open circles and black dots are the experimental points for the first and second warming-up respectively.

by a significant decrease in the absolute value of the electrical conductivity in the temperature interval of $60-300 \mathrm{~K}$. Subsequent cooling cycles affected the shape of $\sigma(T)$ curves much less.

The changes in $\sigma(T)$ are accompanied by changes in $R_{\mathrm{H}}(T)$. Figure 2 shows the dependence for sample No. 354. It is seen that after the second warming up, the inversion point is shifted towards higher temperatures.

\section{Discussion}

The objects under consideration differ basically from both $\mathrm{SnTe}$ and $\mathrm{PbTe}$ bulk and thin film samples. The superlattice consists of layers of materials one of which $(\mathrm{SnTe})$ does undergo and other $(\mathrm{PbTe})$ does not undergo the structural phase transition. This should determine the special influence of the phase transition on the temperature dependencies of the SL properties.

When the critical phase transition temperature is reached, there should appear additional shear strains at the interface, which can be released by the defect formation. It should be mentioned that in the vicinity of the structural phase transition the defect formation demands less energy than in the temperature region away from the instability point $[6,7]$. The reversible structural changes in SnTe layers lead to irreversible changes in electrical properties of the SL's due to defect formation. The idea of defect formation on the interfaces under the phase transition is quite consistent with the fact that the temperature dependence of electrical conductivity changes irreversibly after the very first cooling cycle. Since defects are additional centres of carrier scattering, one should expect a drop in the electrical conductivity of the SL, which is observed in the experiments.

It should be pointed out that phase transition occurs only for relatively small hole concentration, and the critical temperature for the phase transition increases 
with the decrease in the concentration [5]. For the highest hole concentration, the phase transition is suppressed. In Ref. [8], it was concluded that in the $\mathrm{PbTe} / \mathrm{SnTe}$ SLs, the hole concentrations in the SnTe layers were considerably smaller than those in the single films and bulk crystals of SnTe.

It is interesting to compare the temperature dependencies of electrical conductivity of the SL's obtained under the same epitaxial conditions and having the same parameters such as period length and period number but differing by growth orientation. Two such samples, Nos. 364 and 354, are compared in Fig. 1.

As it is seen for the (111) SL's the anomaly in $\sigma(T)$ does not appear at all and $\sigma(T)$ dependence remains unchanged in all the cooling cycles.

The difference in these objects behaviour can be explained as follows. The displacing phase transition in SnTe layer means shift of the crystallographic planes which contain Sn and Te along the axis $\langle 111\rangle$ and then phase transition in the (111) $\mathrm{PbTe} / \mathrm{SnTe} \mathrm{SL}$ is not accompanied by defect formation, because of absence of shear strains on the interface in contrast to (001) $\mathrm{PbTe} / \mathrm{SnTe} \mathrm{SL}$ 's. In the result of that, the effects accompanying the phase transition in the SL constituents can be considerable and on the interface they should be taken into account on working out and optimization of the SL growth methods.

\section{Acknowledgment}

This work was partly supported by Research Project BW 62-116.

\section{References}

[1] K. Murase, S. Ishida, S. Takaoka, T. Okimura, Surf. Sci. 170, 486 (1986).

[2] O.A. Mironov, S.N. Chistyakov, I.Yu. Skrylev, A.I. Fedorenko, A.Yu. Sipatov, B.A. Savitskii, L.P. Shpakovskaya, O.N. Nashchekina, M. Oszwałdowski, Superlattices Microstruct. 8, 361 (1990).

[3] O.A. Mironov, S.N. Chistyakov, A.I. Fedorenko, L.P. Shpakovskaya, A.Yu. Sipatov, B.A. Sa vitskii, O.N. Nashchekina, M. Oszwałdowski, Acta Phys. Pol. A 80, 329 (1991).

[4] O.A. Mironov, B.A. Sa vitskii, A.Yu. Sipatov, A.I. Fedorenko, JETP Lett. 48, 100 (1988).

[5] H. Kawamura, Lect. Notes Phys. 133, 470 (1980).

[6] V.I. Litvinov, V.K. Dugaev, K.D. Tovstyuk, Phys. Lett. A 92, 186 (1982).

[7] V.I. Litvinov, Sov. Phys.-Solid State 26, 717 (1984).

[8] V.I. Litvinov, M. Oszwałdowski, Phys. Status Solidi A 145, 503 (1994). 